\title{
Posterior fossa infarct following Viper bite: a paradox
}

Deepu D (1), Hrishikesh S (2), Suma MT (3), Zoya V (1)

(1) Department of Medicine, Christian Medical College and Hospital, Vellore, Tamil Nadu, India; (2) Department of Neurosurgery, Christian Medical College and Hospital, Vellore, Tamil Nadu, India; (3) Department of Anesthesia, Christian Medical College and Hospital, Vellore, Tamil Nadu, India.

\begin{abstract}
Cerebral infarction after a viper bite is relatively uncommon. A combination of factors has been implicated in the pathophysiology of infarct following snakebite. In this case report, the clinical outcome after a posterior circulation infarct and various possibilities that could lead to such a catastrophic event are discussed. The present study stresses the need to keep hydration, blood pressure and central venous pressure optimal in all snakebite patients. Cerebral infarction should be considered a differential diagnosis, in any patient with neurological deterioration following snakebite. Prognosis of such patients with posterior circulation stroke remains poor and decompressive craniectomy has not been found to be helpful.
\end{abstract}

Key words: snakebite, cerebral infarction, Russell's viper, posterior cranial fossa.

\section{INTRODUCTION}

Approximately 35,000 to 50,000 deaths are attributed to snakebites in India each year according to the World Health Organization (WHO) (1). Neurological sequelae are more often due to subarachnoid or intracerebral hemorrhage and rarely due to cerebral infarction (2). The pathophysiology of the latter is unknown(3). Herein, we describe a 48-year-old woman envenomed by a Russell's viper who developed posterior circulation stroke.

\section{CASE REPORT}

A previously healthy 48-year-old woman was brought to our emergency ward 20 hours following snakebite, with progressively worsening sensorium and respiratory distress. The snake, which had been killed after the bite, was also brought to the hospital by the patient's relatives, and was identified as a Russell's viper (Daboia russelii). During examination, the patient's Glasgow coma scale (GCS) was found to be $10 / 15$ with no focal neurological deficits. There was local inflammation and swelling over the right hand and forearm near the bite site, on which magnesium sulfate dressings were applied. She was intubated and ventilated for impending respiratory arrest. The possibility of an intracranial bleed was considered because of her low sensorium. A computerized tomography (CT) scan was therefore done, which, however, revealed a large infarct involving right cerebellar hemisphere, medulla and pons with mass effect and obstructive hydrocephalus (Figure 1).

Laboratory investigations showed hypernatremia, leukocytosis with neutrophilic predominance, normal coagulation profile and platelet counts. She underwent a decompressive craniectomy and excision of the posterior arch of $\mathrm{C} 1$ vertebra. Intraoperatively, the cerebellum was noted to be pale and bulging. There was no hypoxia or hypotension during anesthesia and surgery. Postoperatively, she was managed with antibiotics (piperacillin/tazobactam), ventilation 


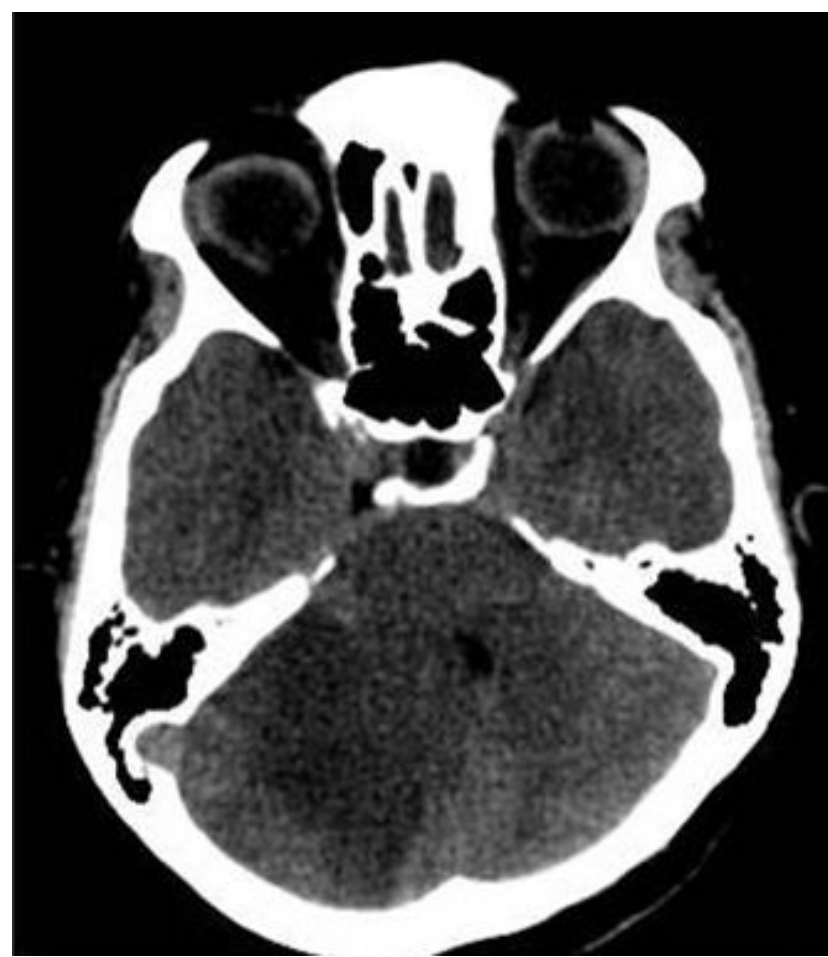

Figure 1. Brain CT with an axial section through the posterior fossa showing a large infarct involving the right cerebellar hemisphere, medulla, pons and resultant mass effect on the fourth ventricle.

and antiedema measures (20\% mannitol) for 48 hours.

After stopping sedation, GCS score remained $2 \mathrm{~T} / 15$. Repeat CT scan revealed adequate bony decompression of the posterior fossa; however, there was no change in mass effect on the brain stem. Since there was no improvement in her condition, the patient's immediate relatives wanted her to be discharged from the hospital, after having explained the poor prognosis. Therefore, she was discharged on request and taken home.

\section{DISCUSSION}

Cerebral infarcts following viper envenomation, especially involving the posterior fossa, are very rare. Lee et al. (4) described a fatal case of pontine infarct following proximal basilar artery occlusion after viper bite. Mugundhan et al. (2) reported a fatal case with right occipital and bilateral cerebellar infarct as a result of posterior circulation stroke. In addition, supratentorial infarcts reportedly have a better clinical outcome compared to infratentorial infarcts (3).
Several hypotheses attempt to explain the occurrence of infarcts following viper envenomation. Hypotension was documented in all series as one of the causes of infarcts (3, 5). This is due to vasodilatation and loss of vasomotor tone provoked by the viper toxin. Russell's viper toxin has been shown to have both procoagulant and anticoagulant properties $(3,6)$. Arginine esterase hydrolases present in viper venom are known to aggregate platelets. Activation of procoagulant pathway can lead to intraluminal thrombus formation. As in early disseminated intravascular coagulopathy (DIC), direct action of toxins causes vascular endothelial damage, with probable release of vascular endothelial growth factor and von Willebrand's factor that also promote thrombus formation and subsequent infarcts (3). Another possibility is a preexisting procoagulant state, due to a mutation in factor $\mathrm{V}$, deficiencies of proteins $\mathrm{C}$ or $\mathrm{S}$, or antithrombin III or antiphospholipid antibodies (5). Direct cardiotoxic effect of viper venom leading to dysrhythmias, which provoke cardiac thromboembolism, also has been implicated in ischemic sequelae (5).

A combination of factors could have caused the infarction in our patient. The presence of local inflammation, elevated total leucocyte counts and neutrophilic shift, even though there was no fever, may have been an indication of early sepsis. Along with infection, delayed treatment and dehydration could have lead to hypoperfusion and increased blood viscosity.

Involvement of the posterior circulation could be due to a congenital anomaly such as hypoplastic vertebral artery or fetal posterior communicating artery; however, no angiogram was done to prove this. No survivors have been reported in the literature of patients with posterior circulation stroke and snakebite, even after a decompressive craniectomy.

This case report emphasizes the need to keep hydration, blood pressure and central venous pressure optimal in any snakebite patient. In case of local infection, it should be managed vigorously with appropriate antibiotics and antiseptic dressings, especially if there is an open wound. Presence of normal coagulation profile and platelet count may mask initial stages of DIC. Neurological status should be closely monitored and if it deteriorates, cerebral infarct should also be considered. 


\section{CONCLUSIONS}

Cerebral infarction can be one of the differential diagnosis of neurological deterioration following Russell's viper bite. Prognosis of such patients with posterior circulation stroke remains poor and decompressive craniectomy has not been found to be helpful.

\section{COPYRIGHT}

(c) CEVAP 2011

\section{SUBMISSION STATUS}

Received: April 18, 2011.

Accepted: May 26, 2011.

Abstract published online: May 30, 2011.

Full paper published online: August 31, 2011.

\section{CONFLICTS OF INTEREST}

There is no conflict.

\section{CORRESPONDENCE TO}

DEEPU DAVID, Department of Medicine II, Christian Medical College and Hospital, Vellore, 632004, Tamil Nadu, India. Phone: +91 416228 203. Mobile: +91 99940 68478. Email: deepudavid@gmail.com.

\section{REFERENCES}

1. WHO/SEARO. Guidelines for the clinical management of snake bites in the Southeast Asian region. Southeast Asian J Trop Med Publ Health. 1999;30(suppl 1):1-85.

2. Mugundhan K, Thruvarutchelvan K, Sivakumar S. Posterior circulation stroke in a young male following snake bite. J Assoc Physicians India. 2008;56:713-4.

3. Boviatsis EJ, Kouyialis AT, Papatheodorou G, Gavra M, Korfias S, Sakas DE. Multiple hemorrhagic brain infarcts after viper envenomation. Am J Trop Med Hyg. 2003;68(2):253-7.

4. Lee BC, Hwang SH, Bae JC, Kwon SB. Brainstem infarction following Korean viper bite. Neurology. 2001;56(9):1244-5.

5. Malbranque S, Piercecchi-Marti MD, Thomas L, Barbey C, Courcier D, Bucher B, et al. Fatal diffuse thrombotic microangiopathy after a bite by the "fer-delance" pit viper (Bothrops lanceolatus) of Martinique. Am J Trop Med Hyg. 2008;78(6):856-61.

6. Ameratunga B. Middle cerebral occlusion following Russel's viper bite. J Trop Med Hyg. 1972;75(5):95-7. 